\title{
EIGENVALUES, GLOBAL BIFURCATION \\ AND POSITIVE SOLUTIONS \\ FOR A CLASS OF NONLOCAL ELLIPTIC EQUATIONS
}

\author{
GUOWEI DAI
}

ABSTRACT. In this paper, we shall study global bifurcation phenomenon for the following Kirchhoff type problem:

$$
\begin{cases}-\left(a+b \int_{\Omega}|\nabla u|^{2} d x\right) \Delta u=\lambda u+h(x, u, \lambda) & \text { in } \Omega, \\ u=0 & \text { on } \Omega .\end{cases}
$$

Under some natural hypotheses on $h$, we show that $\left(a \lambda_{1}, 0\right)$ is a bifurcation point of the above problem. As an application of the above result, we shall determine the interval of $\lambda$, in which there exist positive solutions for the above problem with $h(x, u ; \lambda)=\lambda f(x, u)-\lambda u$, where $f$ is asymptotically linear at zero and asymptotically 3 -linear at infinity. To study global structure of bifurcation branch, we also establish some properties of the first eigenvalue for a nonlocal eigenvalue problem. Moreover, we provide a positive answer to an open problem involving the case $a=0$.

2010 Mathematics Subject Classification. 35B20, 35B32, 35H99, 35R15.

Key words and phrases. Bifurcation; eigenvalue; Kirchhoff type equation; positive solutions.

Research supported by NNSF of China (No. 11261052, 11401477), the Fundamental Research Funds for the Central Universities (No. DUT15RC(3)018) and Scientific Research Project of the Higher Education Institutions of Gansu Province (No. 2014A-009). 


\section{Introduction}

In this paper, we study global bifurcation phenomenon for the following problem:

$$
\begin{cases}-\left(a+b \int_{\Omega}|\nabla u|^{2} d x\right) \Delta u=\lambda u+h(x, u, \lambda) & \text { in } \Omega \\ u=0 & \text { on } \Omega,\end{cases}
$$

where $\Omega$ is a bounded domain in $\mathbb{R}^{N}$ with a smooth boundary $\partial \Omega, a, b>0$ are real constants, $\lambda$ is a parameter and $h: \Omega \times \mathbb{R}^{2} \rightarrow \mathbb{R}$ satisfies the Carathéodory condition in the first two variable and

$$
\lim _{s \rightarrow 0} \frac{h(x, s, \lambda)}{s}=0
$$

uniformly for almost every $x \in \Omega$ and $\lambda$ on bounded sets. Moreover, we assume that $h$ satisfies the following growth restriction:

(G) there exist $c>0$ and $p \in\left(1,2^{*}\right)$ such that

$$
|h(x, s, \lambda)| \leq c\left(1+|s|^{p-1}\right)
$$

for almost every $x \in \Omega$ and $\lambda$ on bounded sets, where

$$
2^{*}= \begin{cases}\frac{2 N}{N-2} & \text { if } N>2 \\ +\infty & \text { if } N \leq 2\end{cases}
$$

Problem (1.1) is related to the stationary problem of a model introduced by Kirchhoff in 1883 to describe transversal oscillations of a stretched string [27]. More precisely, Kirchhoff proposed a model given by the equation

$$
\rho \frac{\partial^{2} u}{\partial t^{2}}-\left(\frac{\rho_{0}}{h}+\frac{E}{2 L} \int_{0}^{L}\left|\frac{\partial u}{\partial x}\right|^{2} d x\right) \frac{\partial^{2} u}{\partial x^{2}}=f(x, u),
$$

where $\rho, \rho_{0}, h, E, L$ are constants, $f$ is an external force, which extends the classical D'Alembert's wave equation by considering the effect of changing in length of the string during vibration. Problem (1.1) received much attention only after Lions [31] proposed an abstract framework to the problem. Some important and interesting results can be found, for example, in [2], [4], [15], [14], [26]. Recently, many mathematicians were studying problem (1.1) by variational methods, see e.g. [5], [6], [32], [33], [35], [38], [41] and references therein.

The authors of [28] studied problem (1.1) with $h(x, s, \lambda)=\lambda f(x, s)-\lambda s$ by using the topological degree argument and variational method. Under some assumptions on $f$, they provided a positive answer to the existence of positive solutions to (1.1) for the cases $a, b>0$ and $a>0, b=0$. They pointed out that the case $a=0$ and $b>0$ is an open problem. The study of Kirchhoff type 
equations has also been extended to the case involving the $p$-Laplacian and $p(x)$ Laplacian. We refer the readers to [3], [7]-[11], [17], [18], [20] for an overview and references on this subject.

A distinguishing feature of problem (1.1) is that the first equation contains a nonlocal coefficient $a+b \int_{\Omega}|\nabla u|^{2} d x$, and hence the equation is no longer a pointwise identity. Moreover, the first equation of problem (1.1) with $h \equiv 0$ is not homogeneous. So problem (1.1) is a fully nonlinear problem what raises some essential difficulties to study this kind of problems.

The main aim of this paper is to establish the global bifurcation result for (1.1) and to study its applications. Let $\lambda_{1}$ denote the first eigenvalue of the following problem:

$$
\begin{cases}-\Delta u=\lambda u & \text { in } \Omega \\ u=0 & \text { on } \Omega .\end{cases}
$$

It is well known that $\lambda_{1}$ is simple, isolated and it is the unique principal eigenvalue of (1.3). The first result of this paper is the following theorem.

THEOREM 1.1. The pair $\left(a \lambda_{1}, 0\right)$ is a bifurcation point of (1.1). Moreover, there is a component $\mathcal{C}$ of the set of nontrivial solutions of (1.1) in $\mathbb{R} \times H_{0}^{1}(\Omega)$ whose closure contains $\left(a \lambda_{1}, 0\right)$ and it is either unbounded or contains a pair $(a \bar{\lambda}, 0)$ for some $\bar{\lambda}$, an eigenvalue of (1.3) with $\bar{\lambda} \neq \lambda_{1}$.

As is known, this result is proved in the case $a=1$ and $b=0$ (see [36]). It has also been extended to the $p$-Laplacian problem in the case $a=1$ and $b=0$ (see [16]). While, (1.1) is a fully nonlinear problem. So the Rabinowitz global bifurcation theorem cannot be used directly to obtain our result. We shall transfer problem (1.1) into a new form and then use the Rabinowitz global bifurcation theorem to prove Theorem 1.1 in Section 2.

In Section 3 we study the component $\mathcal{C}$, obtained in Theorem 1.1 , for the following eigenvalue problem:

$$
\begin{cases}-\left(\int_{\Omega}|\nabla u|^{2} d x\right) \Delta u=\mu u^{3} & \text { in } \Omega, \\ u=0 & \text { on } \partial \Omega .\end{cases}
$$

Let

$$
\mu_{1}=\inf \left\{\left(\int_{\Omega}|\nabla u|^{2} d x\right)^{2}: u \in H_{0}^{1}(\Omega), \int_{\Omega} u^{4} d x=1\right\} .
$$

We recall that a nodal domain of $u$ is a connected component of $\Omega \backslash\{x \in \Omega$ : $u(x)=0\}$. Our second result is

TheOREM 1.2. Assume that $N \leq 3$. Then $\mu_{1}>0$ is the first eigenvalue of (1.4) and it has the following properties: 
(a) Any eigenfunction $u$ corresponding to $\mu_{1}$ belongs to $C^{1, \alpha}(\bar{\Omega})$ for some $\alpha \in(0,1)$, and $\partial u(x) / \partial \gamma<0$ if $u$ is nonnegative, where $\gamma$ is the outer unit normal at $x \in \partial \Omega$.

(b) $\mu_{1}$ is the principal eigenvalue of (1.4).

(c) $\mu_{1}$ is simple if one of the following is satisfied:

(i) $\Omega$ is an open ball in $\mathbb{R}^{N}$;

(ii) $\Omega \subseteq \mathbb{R}^{2}$ is symmetric in $x$ and $y$, and convex in $x$ and $y$ directions; or

(iii) $\Omega \subseteq \mathbb{R}^{2}$ is convex.

(d) If one of conditions in (c) is satisfied, (1.4) has a positive solution if and only if $\mu=\mu_{1}$.

(e) Let $v$ be any eigenfunction associated to an eigenvalue $\mu>\mu_{1}$ and $\mathcal{N}$ be its any nodal domain. Then we have

$$
|\mathcal{N}| \geq c,
$$

where $c>0$ is some constant depending only on $N$ and $\mu$.

(f) If one of conditions in (c)is satisfied, then $\mu_{1}$ is isolated.

The authors of [35] proved the existence of $\mu_{1}$. In [28], the authors proved parts (c) and (d) in the case when $\Omega$ is a ball. Recently, the authors of [29] showed parts (b)-(d). To the best of our knowledge, properties (a), (e) and (f) are the first results on this kind of problems. So the results of Theorem 1.2 improve and generalize the corresponding results of [28], [29] and [35].

In Section 4, we describe the component $\mathcal{C}$ for problem $(1.1)$ with $h(x, s, \lambda)=$ $\lambda f(x, s)-\lambda s$, i.e. for the following problem:

$$
\begin{cases}-\left(a+b \int_{\Omega}|\nabla u|^{2} d x\right) \Delta u=\lambda f(x, u) & \text { in } \Omega \\ u=0 & \text { on } \Omega\end{cases}
$$

where $\Omega$ satisfies one of conditions in Theorem $1.2(\mathrm{c})$. We assume that $f$ satisfies the following conditions:

(f1) $f: \Omega \times \mathbb{R} \backslash \mathbb{R}^{-} \rightarrow \mathbb{R} \backslash \mathbb{R}^{-}$is a Carathéodory function such that $f(x, s) s>0$ for almost every $x \in \Omega$ and any $s>0$.

(f2) There exists $f_{0} \in(0,+\infty)$ such that

$$
\lim _{s \rightarrow 0^{+}} \frac{f(x, s)}{a \lambda_{1} s}=f_{0}
$$

uniformly with respect to almost every $x \in \Omega$.

(f3) There exists $f_{\infty} \in(0,+\infty)$ such that

$$
\lim _{s \rightarrow+\infty} \frac{f(x, s)}{b \mu_{1} s^{3}}=f_{\infty}
$$

uniformly with respect to almost every $x \in \Omega$. 
Our third result is

TheOREM 1.3. Assume that $N \leq 3, \Omega$ satisfies one of conditions in Theorem $1.2(\mathrm{c})$ and $f$ satisfies (f1)-(f3). Then $\left(1 / f_{0}, 0\right)$ is the unique bifurcation point of the set of positive solutions of (1.6). Moreover, there is an unbounded component $\mathcal{C}$ in $\mathbb{R} \times H_{0}^{1}(\Omega)$ whose closure links $\left(1 / f_{0}, 0\right)$ to $\left(1 / f_{\infty}, \infty\right)$.

REMARK 1.4. The reason of restriction $N \leq 3$ in Theorem 1.2 is to ensure $u^{3} \in L^{2}(\Omega)$ for any $u \in H_{0}^{1}(\Omega)$ which is crucial for our proof. If $N=4$, the authors of [29] have showed that $\mu_{1}>0$ but it cannot be achieved in $H_{0}^{1}(\Omega)$. If $N \geq 5$, they also showed that $\mu_{1}=0$. In addition, we pose the condition $N \leq 3$ as our proof depends Theorem 1.2 and the fact $4<2^{*}$.

REMARK 1.5. Note that Theorem 1.1 of [28] is our corollary of Theorem 1.3 if $\Omega$ satisfies one of conditions in Theorem $1.2(\mathrm{c})$. We also note that Theorem 1.2 (ii) (with $a>0$ ) of [28] is the corollary of Theorem 1.3 if $\Omega$ satisfies one of conditions in Theorem 1.2(c). Clearly, Theorem 1.2 (i) of [28] does not occur because $\left(1 / f_{\infty}, \infty\right)$ is the unique bifurcation point of positive solutions set of (1.6) where a bifurcation from infinity occurs. Moreover, our assumptions on $f$ are more concise and weaker than the corresponding ones of $[28$, Theorems 1.1 , $1.2,5.1$ and 5.2$]$.

REMARK 1.6. From Theorem 1.3, we can see that any positive solution of (1.6) lies in $\mathcal{C}$. That is to say, we find the range of all positive solutions. So one only needs to study the structure of $\mathcal{C}$ to find the positive solution of (1.6).

REMARK 1.7. By standard elliptic regularity theory (see [21], [22]), we know that any weak solution of (1.1) or (1.6) belongs to $C^{1, \alpha}(\bar{\Omega})$ with $\alpha \in(0,1)$ under conditions (1.2) and (G) or (f1)-(f3).

In Section 5, we consider the case $a=0$ in (1.6) and give a positive answer to the above mentioned open problem. Concretely, we pose the following assumption on $f$ :

(f4) there exists $\widetilde{f}_{0} \in(0,+\infty)$ such that

$$
\lim _{s \rightarrow 0^{+}} \frac{f(x, s)}{\lambda_{1} s}=\widetilde{f}_{0}
$$

uniformly with respect to almost every $x \in \Omega$.

Our last result is the following theorem.

THEOREM 1.8. Assume that $a=0, N \leq 3$, $\Omega$ satisfies one of conditions in Theorem 1.2 (c) and $f$ satisfies (f1), (f3) and (f4). Then $(0,0)$ is the unique bifurcation point of the set of positive solutions of (1.6). Moreover, there is an unbounded component $\mathcal{C}$ in $\mathbb{R} \times H_{0}^{1}(\Omega)$ whose closure links $(0,0)$ to $\left(1 / f_{\infty}, \infty\right)$. 
REMARK 1.9. From Theorem 1.8, we can easily see that (1.6) has a positive solution with $\lambda=1$ and $f_{\infty}<1$. This gives a positive answer to an open problem proposed by the authors in [28].

REMARK 1.10. Note that $f$ is asymptotically linear at zero in Theorem 1.8 which is different from [28]. In [28], $f$ is assumed to be asymptotically 3-linear at zero in the case $a=0$. We shall consider this situation in our future work.

The last section concludes the paper and outlines our future work.

We end this section by introducing some notation conventions which will be used later in this paper. Let $X$ be the usual Sobolev space $H_{0}^{1}(\Omega)$ with the norm $\|u\|=\left(\int_{\Omega}|\nabla u|^{2} d x\right)^{1 / 2}$ and $X^{*}$ be its dual space. Denote by $\langle\cdot, \cdot\rangle$ the duality pairing between $X$ and $X^{*}$. We write $u_{n} \rightarrow u$ and $u_{n} \rightarrow u$ for the weak and strong convergence of the sequence $\left\{u_{n}\right\}$ in $X$, respectively. Use $q^{\prime}=q /(q-1)$ to denote the conjugative number of $q$ with $q>1$. For a measurable set $A$ of $\mathbb{R}^{N}$ we denote its measure by $|A|$. Also, we denote by $c$ and $c_{i}, i \in \mathbb{N}$, generic positive constants (the exact value may be different from line to line).

\section{Global bifurcation}

Firstly, consider the following auxiliary problem:

$$
\begin{cases}-\Delta u=f(x) & \text { in } \Omega, \\ u=0 & \text { on } \partial \Omega .\end{cases}
$$

As is known, problem (2.1) possesses a unique weak solution for each $f \in X^{*}$. Let us denote by $G(f)$ the unique weak solution of (2.1). Then $G: X^{*} \rightarrow X$ is a linear continuous operator. Since the embedding $X \hookrightarrow L^{q}(\Omega)$ is compact for each $q \in\left(1,2^{*}\right)$, the restriction of $G$ to $L^{q^{\prime}}(\Omega)$ is a completely continuous operator.

Clearly, the pair $(\lambda, u)$ is a solution of (1.1) if and only if $(\lambda, u)$ satisfies

$$
u=G\left(\frac{1}{a+b\|u\|^{2}}(\lambda u+H(\lambda, u))\right),
$$

where $H(\lambda, \cdot)$ denotes the usual Nemitskiŭ operator associated with $h$. From condition $(\mathrm{G})$ and $2<2^{*}$, we can see that $G: L^{p^{\prime}}(\Omega) \cup L^{2}(\Omega) \rightarrow X$ is completely continuous.

Proof of Theorem 1.1. Let

$$
L u=\frac{1}{a} G(u), \quad \widetilde{H}(\lambda, u)=\frac{1}{a+b\|u\|^{2}} G(H(\lambda, u))-\frac{\lambda b\|u\|^{2}}{a\left(a+b\|u\|^{2}\right)} G(u) .
$$

Clearly, $L: X \rightarrow X$ is linear and completely continuous operator, $\widetilde{H}: \mathbb{R} \times X \rightarrow X$ is compact. Moreover, it is easy to see that $a \lambda_{1}$ is a simple characteristic value 
of $L$. Then equation $(2.2)$ is equivalent to

$$
u=\lambda L u+\widetilde{H}(\lambda, u) .
$$

Next, we show that $\widetilde{H}=o(\|u\|)$ at $u=0$ uniformly on bounded $\lambda$ intervals. It is sufficient to show that

$$
\lim _{\|u\| \rightarrow 0} \frac{H(x, u)}{\|u\|}=0 \quad \text { in } L^{p^{\prime}}(\Omega) .
$$

Without loss of generality, we may assume that $p>2$. Otherwise, we can consider $\tilde{p}=c p, c>1$ such that $\tilde{p} \in\left(2,2^{*}\right)$. From $p<2^{*}$, we can see that

$$
\frac{p^{\prime}(p-2)}{2^{*}}<\frac{2^{*}-p^{\prime}}{2^{*}} \text {. }
$$

So we can choose a real number $r>1$ such that

It follows that

$$
\frac{p^{\prime}(p-2)}{2^{*}} \leq \frac{1}{r} \leq \frac{2^{*}-p^{\prime}}{2^{*}}
$$

$$
p^{\prime} r(p-2) \leq 2^{*} \quad \text { and } \quad p^{\prime} r^{\prime} \leq 2^{*} .
$$

For any $\varepsilon>0$, in view of (1.2) and $(\mathrm{G})$, we can choose positive numbers $\delta=\delta(\varepsilon)$ and $M=M(\delta)$ such that for almost every $x \in \Omega$, the following relations hold:

$$
\begin{array}{ll}
\left|\frac{h(x, s, \lambda)}{s}\right| \leq \varepsilon & \text { for } 0<|s| \leq \delta, \\
\left|\frac{h(x, s, \lambda)}{s}\right| \leq M|s|^{p-2} & \text { for }|s|>\delta .
\end{array}
$$

Then

$$
\int_{\Omega}\left|\frac{H(\lambda, u)}{u}\right|^{p^{\prime} r} d x \leq \varepsilon|\Omega|+M^{p^{\prime} r} \int_{\Omega}|u|^{p^{\prime} r(p-2)} d x .
$$

From this inequality, (2.3) and $u \rightarrow 0$ in $X$, we get

$$
\left|\frac{H(\lambda, u)}{u}\right|^{p^{\prime}} \rightarrow 0 \quad \text { in } L^{r}(\Omega) .
$$

Let $v=u /\|u\|$. By the boundedness of $v$ in $X,(2.3)$ and continuity of the embedding $X \hookrightarrow L^{2^{*}}(\Omega)$, we have

$$
\int_{\Omega}|v|^{p^{\prime} r^{\prime}} d x \leq c
$$

for some constant $c>0$. Then from (2.4), (2.5) and the Hölder inequality, we obtain

$$
\begin{aligned}
\int_{\Omega}\left|\frac{H(\lambda, u)}{\|u\|}\right|^{p^{\prime}} d x & =\int_{\Omega}\left|\frac{H(\lambda, u)}{|u|}\right|^{p^{\prime}}|v|^{p^{\prime}} d x \\
& \leq\left(\int_{\Omega}\left|\frac{H(\lambda, u)}{u}\right|^{p^{\prime} r} d x\right)^{1 / r}\left(\int_{\Omega}|v|^{p^{\prime} r^{\prime}} d x\right)^{1 / r^{\prime}} \rightarrow 0 .
\end{aligned}
$$


Now, from global bifurcation theory (see [37, Theorem 1.3]), we get the existence of a global branch of the set of nontrivial solution of (1.1) emanating from $\left(a \lambda_{1}, 0\right)$

\section{Properties of the first eigenvalue of a nonlocal problem}

In order to study properties of the component $\mathcal{C}$, obtained in Theorem 1.1, we shall consider the following eigenvalue problem:

$$
\begin{cases}-\left(\int_{\Omega}|\nabla u|^{2} d x\right) \Delta u=\mu u^{3} & \text { in } \Omega, \\ u=0 & \text { on } \partial \Omega .\end{cases}
$$

Let

$$
I(u)=\|u\|^{4}, \quad u \in S:=\left\{u \in X: \int_{\Omega} u^{4} d x=1\right\} .
$$

Denote by $\mathcal{A}$ the class of closed symmetric subsets of $S$, let

$$
\mathcal{F}_{m}=\{A \in \mathcal{A}: i(A) \geq m-1\},
$$

where $m$ is a positive integer and $i(A)$ denotes the Yang index of $A$. The authors of [35] have proved that problem (3.1) possesses unbounded sequences of minimax eigenvalues $0<\mu_{1} \leq \mu_{2}<\ldots$ such that

$$
\mu_{m}=\inf _{A \in \mathcal{F}_{m}} \max _{u \in A} I(u) .
$$

In particular, if $m=1$, taking $A=\{u,-u: u \in S\}$, we get

$$
\mu_{1}=\inf \left\{\|u\|^{4}: u \in X, \int_{\Omega} u^{4} d x=1\right\} .
$$

Next, we are going to study the properties of $\mu_{1}$. These properties are important in the study of global bifurcation phenomena.

Proposition 3.1. Let $u$ be any eigenfunction corresponding to $\mu_{1}$. Then $u \in C^{1, \alpha}(\bar{\Omega})$ for some $\alpha \in(0,1)$. Furthermore, $\partial u(x) / \partial \gamma<0$ if $u$ is nonnegative, where $\gamma$ is the outer unit normal at $x \in \partial \Omega$.

Proof. Note that problem (3.1) is homogeneous. So by scaling we may suppose that $\|u\|=1$. It follows that

$$
\begin{cases}-\Delta u=\mu_{1} u^{3} & \text { in } \Omega, \\ u=0 & \text { on } \partial \Omega .\end{cases}
$$

By the embedding $X \hookrightarrow L^{2^{*}}$ and $N \leq 3$, we have $\mu_{1} u^{3}(\cdot):=f(\cdot) \in L^{2}(\Omega)$. By [25, Theorem 8.12], we know that $u \in W^{2,2}(\Omega)$. Furthermore, by the general Sobolev embedding theorem [19, p. 270], we get $u \in C^{\gamma}(\bar{\Omega})$ for some $\gamma \in(0,1)$. Moreover, by the definition of weak derivative, $\nabla f=\mu_{1} 3 u^{2} \nabla u$. It is easy to verify that $\nabla f \in L^{2}(\Omega)$. So we have $f \in W^{1,2}(\Omega)$. By [25, Theorem 8.13], we 
know that $u \in W^{3,2}(\Omega)$. Again using the general Sobolev embedding theorem [19, p. 270], we get $u \in C^{1, \alpha}(\bar{\Omega})$ for some $\alpha \in(0,1)$. Furthermore, if $u \geq 0$, by the strong maximum principle [23, Theorem 1.2], $\partial u(x) / \partial \gamma<0$ for all $x \in \partial \Omega$.

Proposition 3.2. Let $u$ be an eigenfunction associated with $\mu_{1}$, then either $u>0$ or $u<0$ in $\Omega$, i.e. $\mu_{1}$ is the principal eigenvalue of (3.1).

Proof. We notice that if $u$ is an eigenfunction, so is $v:=|u|$. Without loss of generality, we shall assume that $\|v\|=1$. So we have

$$
\begin{cases}-\Delta v=\mu_{1} v^{3} & \text { in } \Omega, \\ v=0 & \text { on } \partial \Omega .\end{cases}
$$

By the strong maximum principle [25, Theorem 8.19], we know that $v>0$ in the whole domain. By the continuity of $u$, either $u$ or $-u$ is positive in the whole domain.

Proof of Theorem 1.2. Propositions 3.1 and 3.2 imply properties (a) and (b), respectively. So we only need to prove properties (c)-(f). Although property (c) was proved in [28], [29], we shall still give a rough sketch of the proof here for reader's convenience.

(c) Consider the following auxiliary problem:

$$
\begin{cases}-\Delta u=u^{3} & \text { in } \Omega, \\ u=0 & \text { on } \partial \Omega .\end{cases}
$$

If $\Omega$ is an open ball in $\mathbb{R}^{N}$, Lemma 2.3 of [24] implies that the positive solution of (3.3) is unique. Let $u, v$ be two eigenfunctions associated with $\lambda_{1}$. Then $\sqrt[2]{\lambda_{1}} u /\|u\|$ and $\sqrt[2]{\lambda_{1}} v /\|v\|$ are both solutions to (3.3). Hence $u=\|u\| v /\|v\|$, which shows that $\lambda_{1}$ is simple. If $\Omega \subseteq \mathbb{R}^{2}$ is symmetric in $x$ and $y$, and convex in $x$ and $y$ directions, Theorem 2.1 of [12] and Theorem 5 of [13] imply that the positive solution of (3.3) is unique. Similar argument shows that $\lambda_{1}$ is simple. If $\Omega \subseteq \mathbb{R}^{2}$ is convex, Theorem 1 of [30] implies that the least energy positive solution of (3.3) is unique, where the least energy solution is the one which achieves

$$
\inf _{u \in X, u \neq 0} \frac{\int_{\Omega}|\nabla u|^{2} d x}{\left(\int_{\Omega}|u|^{4} d x\right)^{1 / 2}} .
$$

Suppose that $u$ and $v$ are positive solutions of (1.4) with $\lambda=\lambda_{1}$, then both $u$ and $v$ are least energy positive solutions of (3.3). So $u=c v$ for some constant $c>0$. 
(d) Suppose on the contrary that (3.1) with $\mu>\mu_{1}$ has a positive solution $v$, and let $u$ be a positive eigenfunction corresponding to $\mu_{1}$. Similarly to Proposition 3.1, we can show that $v \in C^{1, \alpha}(\bar{\Omega})$ for some $\alpha \in(0,1)$ and $\partial v(x) / \partial \gamma<0$. By this and Proposition 3.1, there exists a constant $t_{0}>0$ large enough such that $t_{0} v \geq u$. Clearly, $\widetilde{v}:=t_{0} v$ is also an eigenfunction of (3.1). Define $f^{1}$ and $f^{2}$ on $X$ by

$$
f^{1}(w)=\frac{1}{4}\|w\|^{4}, \quad f^{2}(w)=\frac{1}{4} \int_{\Omega} w^{4} d x .
$$

Let $A=\left(f^{1}\right)^{\prime}$ and $B=\left(f^{2}\right)^{\prime}$. Then $w$ is a weak solution of (3.1) if and only if $A w=\mu B w$. It is not difficult to show that $B u \leq B \widetilde{v}$. Then

$$
A u=\mu_{1} B u \leq \mu_{1} B \widetilde{v}=\mu B(\eta \widetilde{v})=A(\eta \widetilde{v}) \quad \text { with } \eta=\left(\frac{\mu_{1}}{\mu}\right)^{1 / 3}<1 .
$$

Taking $\varphi=(u-\eta \widetilde{v})^{+}$as a test function in $A u \leq A(\eta \widetilde{v})$, it follows from (a) of Proposition 5.2, which will be proved in Section 5, that $\nabla(u-\eta \widetilde{v})^{+}=0$, this implies $(u-\eta \widetilde{v})^{+}=0$ and so $u \leq \eta \widetilde{v}$ in $\Omega$. Repeating this argument $n$ times, we obtain that $0 \leq u \leq \eta^{n} \widetilde{v}$. Letting $n \rightarrow+\infty$, we get $u \equiv 0$. This is a contradiction. So $v$ must change sign.

(e) By an argument similar to that of Proposition 3.1, we know that $v \in$ $C(\Omega)$, then $v_{\left.\right|_{\mathcal{N}}} \in H_{0}^{1}(\mathcal{N})$. Define

$$
w= \begin{cases}v & \text { for } x \in \mathcal{N} \\ 0 & \text { for } x \in \Omega \backslash \mathcal{N} .\end{cases}
$$

It is easy to see that $w \in X$. We first consider the case $N=3$. Then

$$
\left(\int_{\mathcal{N}}|\nabla w|^{2} d x\right)^{2}=\mu \int_{\mathcal{N}} w^{4} d x
$$

By the Hölder inequality and the Sobolev embeddings we have

$c^{4}\left(\int_{\mathcal{N}}|w|^{6} d x\right)^{2 / 3} \leq\left(\int_{\mathcal{N}}|\nabla w|^{2} d x\right)^{2}=\mu \int_{\mathcal{N}} w^{4} d x \leq \mu\left(\int_{\mathcal{N}} w^{6} d x\right)^{2 / 3}|\mathcal{N}|^{1 / 3}$, where $c>0$ is the best embedding constant of $H_{0}^{1}(\mathcal{N}) \hookrightarrow L^{6}(\mathcal{N})$. It follows that $|\mathcal{N}| \geq c^{12} / \mu^{3}$. For $N=1$ or 2 , it is well known that $H_{0}^{1}(\mathcal{N})$ continuously embeds in $L^{\infty}(\mathcal{N})$ with the best embedding constant $c>0$. From this fact and reasoning as above, we can show that $|\mathcal{N}| \geq c^{4} / \mu$.

(f) Assume by contradiction that there exists a sequence of eigenvalues $\mu_{n} \in\left(\mu_{1}, \delta\right)$ for some constant $\delta>\mu_{1}$ which converges to $\mu_{1}$. Let $u_{n}$ be the corresponding eigenfunctions. Property (d) implies that $u_{n}$ changes sign. Integration by parts gives

$$
\left(\int_{\Omega}\left|\nabla u_{n}\right|^{2} d x\right)^{2}=\mu_{n} \int_{\Omega}\left(u_{n}\right)^{4} d x .
$$


Define

$$
v_{n}:=\frac{u_{n}}{\left(\int_{\Omega}\left(u_{n}\right)^{4} d x\right)^{1 / 4}}
$$

Obviously, $v_{n}$ is bounded in $X$ so there exists a subsequence, denoted again by $v_{n}$, and $v \in X$ such that $v_{n} \rightarrow v$ in $X$ and $v_{n} \rightarrow v$ in $L^{4}(\Omega)$. Since the functional $I$ is sequentially weakly lower semi-continuous, we have that

$$
\left(\int_{\Omega}|\nabla v|^{2} d x\right)^{2} \leq \liminf _{n \rightarrow+\infty}\left(\int_{\Omega}\left|\nabla v_{n}\right|^{2} d x\right)^{2}=\liminf _{n \rightarrow+\infty} \mu_{n}=\mu_{1} .
$$

On the other hand, $\int_{\Omega}\left(v_{n}\right)^{4} d x=1$ and $v_{n} \rightarrow v$ in $L^{4}(\Omega)$ imply that $\int_{\Omega} v^{4} d x=1$. It follows that

$$
\left(\int_{\Omega}|\nabla v|^{2} d x\right)^{2} \leq \mu_{1} \int_{\Omega} v^{4} d x
$$

The above inequality and the variational characterization of $\mu_{1}$ imply that

$$
\left(\int_{\Omega}|\nabla v|^{2} d x\right)^{2}=\mu_{1} \int_{\Omega} v^{4} d x
$$

Then Proposition 3.2 implies that $v$ is positive or negative. Without loss of generality, we may assume that $v>0$ in $\Omega$. Since $v_{n} \rightarrow v X$, passing if necessary to a subsequence, we can assume that

$$
\begin{aligned}
& v_{n} \rightarrow v \quad \text { in } L^{q}(\Omega) \text { with } q \in\left(1,2^{*}\right), \\
& v_{n} \rightarrow v \quad \text { in a.e. } \Omega .
\end{aligned}
$$

So we conclude that $\left|\Omega_{n}^{-}\right| \rightarrow 0$, where $\Omega_{n}^{-}$denotes the negative set of $u_{n}$. This contradicts estimate (1.5).

\section{Positive solutions}

In this section we apply Theorems 1.1 and 1.2 to study the existence of positive solutions for (1.6).

Lemma 4.1. Assume (f1)-(f3) hold. Then $\left(1 / f_{0}, 0\right)$ is a bifurcation point of (1.6) and the associated bifurcation branch $\mathcal{C}$ in $\mathbb{R} \times X$ whose closure contains $\left(1 / f_{0}, 0\right)$ is either unbounded or contains a pair $\left(\bar{\lambda} /\left(f_{0} \lambda_{1}\right), 0\right)$ where $\bar{\lambda}$ is an eigenvalue of (1.3) and $\bar{\lambda} \neq \lambda_{1}$.

Proof. Let $\vartheta: \Omega \times \mathbb{R} \backslash \mathbb{R}^{-} \rightarrow \mathbb{R} \backslash \mathbb{R}^{-}$be a Carathéodory function such that

$$
f(x, s)=a \lambda_{1} f_{0} s+\vartheta(x, s)
$$

with

$$
\lim _{s \rightarrow 0^{+}} \frac{\vartheta(x, s)}{a \lambda_{1} s}=0 \quad \text { and } \quad \lim _{s \rightarrow+\infty} \frac{\vartheta(x, s)}{s^{3}}=b \mu_{1} f_{\infty}
$$


uniformly with respect to almost every $x \in \Omega$. From (4.1), we can see that $\lambda \vartheta$ satisfies assumptions of (1.2) and $(\mathrm{G})$. Now, Theorem 1.1 can be applied to get the results of this lemma.

Let $P=\{u \in X: u(x)>0$ for all $x \in \Omega\}$ be the positive cone in $X$.

LEMma 4.2. We have $\mathcal{C} \subseteq\left((\mathbb{R} \times P) \cup\left\{\left(1 / f_{0}, 0\right)\right\}\right)$ and the last alternative of Lemma 4.1 is impossible.

Proof. By the strong maximum principle [25, Theorem 8.19], we know that $u>0$ in the whole domain for any nontrivial solution $(\lambda, u) \in \mathcal{C}$. So we have $\mathcal{C} \subseteq((\mathbb{R} \times P) \cup(\mathbb{R} \times\{0\}))$. Suppose on the contrary that there exists $\left(\lambda_{m}, u_{m}\right) \rightarrow\left(\bar{\lambda} /\left(f_{0} \lambda_{1}\right), 0\right)$ when $m \rightarrow+\infty$ with $\left(\lambda_{m}, u_{m}\right) \in \mathcal{C}, u_{m} \not \equiv 0$ and $\bar{\lambda} \neq \lambda_{1}$. So we have $u_{m} \in P$ for each $m \in \mathbb{N}$. Let $v_{m}:=u_{m} /\left\|u_{m}\right\|$, then $\left(\lambda_{m}, v_{m}\right)$ satisfies

$$
v_{m}=G\left(\frac{\lambda_{m}}{a+b\left\|u_{m}\right\|^{2}}\left(a \lambda_{1} f_{0} v_{m}+\frac{\vartheta\left(\lambda, u_{m}\right)}{\left\|u_{m}\right\|}\right)\right) .
$$

By an argument similar to that of Theorem 1.1, we obtain that for some convenient subsequence $v_{m} \rightarrow v_{0}$ as $m \rightarrow+\infty$. Now $v_{0}$ verifies the equation $-\Delta v=\bar{\lambda} v$ and $\left\|v_{0}\right\|=1$. Hence $v_{0}$ must change its sign, and this is a contradiction. Furthermore, it follows that $\mathcal{C} \subseteq\left(P \cup\left\{\left(1 / f_{0}, 0\right)\right\}\right)$ and $\mathcal{C}$ is unbounded in $\mathbb{R} \times X$.

REMARK 4.3. From the proof of Lemma 4.2 , we can see that $\left(1 / f_{0}, 0\right)$ is the unique bifurcation point of the set of positive solutions of (1.6).

Next, we give a Sturm type comparison theorem.

THEOREM 4.4. Assume that $N \leq 3, g$ and $f_{n}$ are two weight functions such that $g \in C(\bar{\Omega}), f_{n} \in L^{3}(\Omega)$ and $f_{n} \not \equiv g$ almost everywhere in $\Omega$ for any $n$ large enough. Let $u$ be a positive weak solution of

$$
\begin{cases}-\|u\|^{2} \Delta u=g(x) u^{3} & \text { in } \Omega, \\ u=0 & \text { on } \partial \Omega .\end{cases}
$$

Let $v_{n} \in W^{2,1}(\Omega)$ be any solution of

$$
-\|v\|^{2} \Delta v=f_{n}(x) v^{3} \quad \text { in } \Omega .
$$

If $g u^{2} \leq f_{n} v_{n}^{2}$ for almost every $x \in \Omega$ and $n$ is large enough, then $v_{n}$ must change sign for $n$ large enough.

Proof. Without loss of generality, we may assume that $\|u\|=\left\|v_{n}\right\|=1$ for any $n \in \mathbb{N}$. Suppose the contrary, e.g. that $v_{n}>0$ for $n$ large enough. Then by the following Picone's identity:

$$
|\nabla u|^{2}-\nabla\left(\frac{u^{2}}{v}\right) \nabla v=|\nabla u|^{2}+\frac{u^{2}}{v^{2}}|\nabla v|^{2}-2 \frac{u}{v} \nabla u \nabla v \geq 0
$$


and an easy calculation, we obtain, for $n$ large enough,

$$
\begin{aligned}
0 \leq \int_{\Omega}\left(|\nabla u|^{2}+\frac{u^{2}}{v_{n}^{2}}\left|\nabla v_{n}\right|^{2}-2 \frac{u}{v_{n}} \nabla u \nabla\right. & \left.v_{n}\right) d x \\
& =\int_{\Omega}\left(g(x) u^{2}-f_{n}(x) v_{n}^{2}\right) u^{2} d x \leq 0 .
\end{aligned}
$$

Consequently, we have $u=c v_{n}$ for $n$ large enough. Furthermore, we have $u=$ $\pm v_{n}$ for $n$ large enough. But this is impossible since $f_{n} \not \equiv g$ almost everywhere in $\Omega$ for $n$ large enough. This accomplishes the proof.

Proof of Theorem 1.3. In view of Lemmas 4.1, 4.2 and Remark 4.3, it is sufficient to show that $\mathcal{C}$ joins $\left(1 / f_{0}, 0\right)$ to $\left(1 / f_{\infty}, \infty\right)$. Let $\left(\lambda_{n}, u_{n}\right) \in \mathcal{C}$, where $u_{n} \not \equiv 0$ satisfies $\lambda_{n}+\left\|u_{n}\right\| \rightarrow+\infty$. Since $(0,0)$ is the only solution of (1.6) for $\lambda=0$, we have $\mathcal{C} \cap(\{0\} \times X)=\emptyset$. It follows that $\lambda_{n}>0$ for all $n \in \mathbb{N}$.

We claim that there exists a constant $M$ such that $\lambda_{n} \in(0, M]$ for $n \in \mathbb{N}$ large enough. Suppose the contrary, that $\lim _{n \rightarrow+\infty} \lambda_{n}=+\infty$. Since $\left(\lambda_{n}, u_{n}\right) \in \mathcal{C}$, it follows that

$$
\Delta u_{n}+\frac{\lambda_{n}}{a+b\left\|u_{n}\right\|^{2}} \frac{f\left(x, u_{n}\right)}{u_{n}} u_{n}=0 \quad \text { in } \Omega .
$$

We consider two cases to deduce a contradiction.

Case 1. There exists a constant $c>0$ such that $\left\|u_{n}\right\| \leq c$ for $n$ large enough. In this case, we have

$$
\frac{1}{a} \geq \frac{1}{a+b\left\|u_{n}\right\|^{2}} \geq \frac{1}{a+b c^{2}} .
$$

From (f1)-(f3), we can see that $f\left(x, u_{n}\right) / u_{n} \geq \sigma$ for some $\sigma>0$ and almost every $x \in \Omega$ and all $n \in \mathbb{N}$. By (f2) and (f3), $F\left(x, u_{n}\right) / u_{n} \in L^{5 / 3}(\Omega)$, where $F$ denotes the usual Nemitskiu operator associated with $f$. Applying Theorem 2.6 of [1], we have that $u_{n}$ must change its sign in $\Omega$, what contradicts Lemma 4.2.

Case 2. $\left\|u_{n}\right\| \rightarrow+\infty$ as $n \rightarrow+\infty$.

Now, consider

$$
\left\|u_{n}\right\|^{2} \Delta u_{n}+\lambda_{n} \frac{\left\|u_{n}\right\|^{2}}{a+b\left\|u_{n}\right\|^{2}} \frac{f\left(x, u_{n}\right)}{u_{n}^{3}} u_{n}^{3}=0 \quad \text { in } \Omega .
$$

For any fixed $\varepsilon \in(0,1 / b)$, obviously, there exists $N_{1}>0$ such that, for any $n>N_{1}$,

$$
\frac{\left\|u_{n}\right\|^{2}}{a+b\left\|u_{n}\right\|^{2}} \geq \frac{1}{b}-\varepsilon .
$$

By (f1)-(f3), there exists a constant $\varrho>0$ such that $f\left(x, u_{n}\right) / u_{n} \geq \varrho$ for almost every $x \in \Omega$ and $n$ large enough. Let

$$
f_{n}(x)=\lambda_{n} \frac{\left\|u_{n}\right\|^{2}}{a+b\left\|u_{n}\right\|^{2}} \frac{f\left(x, u_{n}(x)\right)}{u_{n}(x)} \frac{1}{u_{n}^{2}(x)} .
$$


Then we have $\lim _{n \rightarrow+\infty} f_{n}(x) u_{n}(x)=+\infty$ for almost every $x \in \Omega$. By (f1)-(f3) and [25, Theorem 8.12], we know that $u_{n} \in W^{2,2}(\Omega)$. So we have $u_{n} \in W^{2,1}(\Omega)$.

On the other hand, by Theorem 1.1 of [23], we have that for any nonempty compact subset $K \subseteq \Omega$, there exists a positive constant $c_{n}$ such that $u_{n} \geq c_{n}$ almost everywhere in $K$. Then it is not difficult to show that $f_{n} \in L^{3}(K)$ for any fixed $n \in \mathbb{N}$. Applying Theorem 4.4 on $K$ with $g(x) \equiv \mu_{1}$, we have that $u_{n}$ must change its sign in $K$ for $n$ large enough. This is a contradiction.

Therefore, we get that $\left\|u_{n}\right\| \rightarrow+\infty$. Let $\varpi: \Omega \times \mathbb{R} \backslash \mathbb{R}^{-} \rightarrow \mathbb{R} \backslash \mathbb{R}^{-}$be a Carathéodory function such that

$$
f(x, s)=b \mu_{1} f_{\infty} s^{3}+\varpi(x, s)
$$

with

$$
\lim _{s \rightarrow+\infty} \frac{\varpi(x, s)}{s^{3}}=0 \text { and } \lim _{s \rightarrow 0^{+}} \frac{\varpi(x, s)}{s}=a \lambda_{1} f_{0}
$$

uniformly with respect to almost every $x \in \Omega$. Then $\left(\lambda_{n}, u_{n}\right)$ satisfies

$$
u_{n}=G\left(\frac{\lambda_{n}}{a+b\left\|u_{n}\right\|^{2}}\left(b \mu_{1} f_{\infty} u_{n}^{3}+\varpi\left(x, u_{n}\right)\right)\right) .
$$

Dividing the above equation by $\left\|u_{n}\right\|$ and letting $\bar{u}_{n}=u_{n} /\left\|u_{n}\right\|$, we get

$$
\bar{u}_{n}=G\left(\frac{\lambda_{n}\left\|u_{n}\right\|^{2}}{a+b\left\|u_{n}\right\|^{2}}\left(b \mu_{1} f_{\infty} \bar{u}_{n}^{3}+\frac{\varpi\left(x, u_{n}\right)}{\left\|u_{n}\right\|^{3}}\right)\right) .
$$

Next, we show

$$
\lim _{n \rightarrow+\infty} \frac{\varpi\left(x, u_{n}(x)\right)}{\left\|u_{n}\right\|^{3}}=0 \quad \text { in } L^{q^{\prime}}(\Omega)
$$

for some $q<2^{*}$. Without loss of generality, we may assume that $q \geq 4$. Otherwise, we can consider $\widetilde{q}=c q, c>1$ such that $\widetilde{q} \in\left[4,2^{*}\right)$.

From (4.4), for any $\varepsilon>0$, we can choose $\delta=\delta(\varepsilon)$ and $M=M(\delta)$ such that for almost every $x \in \Omega$ and any $n \in \mathbb{N}$, the following relations hold:

$$
\begin{array}{ll}
|\varpi(x, s)| \leq M & \text { for }|s| \leq \delta, \\
|\varpi(x, s)| \leq \varepsilon|s|^{3} & \text { for }|s|>\delta .
\end{array}
$$

By (4.6) and (4.7), we can easily show that

$$
\int_{\Omega}\left|\frac{\varpi\left(x, u_{n}(x)\right)}{\left\|u_{n}\right\|^{3}}\right| d x \leq \frac{c}{\left\|u_{n}\right\|^{3 q^{\prime}}}+\varepsilon \int_{\Omega}\left|\bar{u}_{n}\right|^{3 q^{\prime}} d x .
$$

It follows from $q \geq 4$ that $3 q^{\prime}<2^{*}$. From the above inequality, $u_{n} \rightarrow+\infty$ in $X$, $\left\|\bar{u}_{n}\right\|=1$, we get the desired result. It is not difficult to show that $\bar{u}_{n}^{3}$ is bounded in $L^{q^{\prime}}(\Omega)$ and $\lambda_{n}\left\|u_{n}\right\|^{2} /\left(a+b\left\|u_{n}\right\|^{2}\right)$ is bounded in $\mathbb{R}$ for $n$ large enough. By the compactness of $G$, we obtain that $-\|\bar{u}\|^{2} \Delta \bar{u}=\bar{\mu} \mu_{1} f_{\infty} \bar{u}^{3}$, where $\bar{u}=\lim _{n \rightarrow+\infty} \bar{u}_{n}$ and $\bar{\mu}=\lim _{n \rightarrow+\infty} \lambda_{n}$, again choosing a subsequence and relabeling it if necessary. 
It is clear that $\|\bar{u}\|=1$ and $\bar{u} \in \overline{\mathcal{C}} \subseteq \mathcal{C}$ since $\mathcal{C}$ is closed in $\mathbb{R} \times X$. Therefore, Lemma 4.2 implies $\bar{u}>0$. Theorem 1.2 shows that $\bar{\mu}=1 / f_{\infty}$. Therefore, $\mathcal{C}$ joins $\left(1 / f_{0}, 0\right)$ to $\left(1 / f_{\infty}, \infty\right)$.

Corollary 4.5. Assume that $N \leq 3$ and $f$ satisfies (f1)-(f2). Then for

$$
\lambda \in\left(1 / f_{0}, 1 / f_{\infty}\right) \cup\left(1 / f_{\infty}, 1 / f_{0}\right),
$$

problem (1.6) possesses at least one positive solution. In particular, if $f_{0}>1$ $(<1)$ and $f_{\infty}<1(>1)$ then $(1.6)$ possesses at least one positive solution with $\lambda=1$.

ExAmple 4.6. Let $a=b=1, \lambda=1, f(x, s)=\nu s+\mu s^{3}$. By Corollary 4.5, there is an unbounded component $\mathcal{C}$ in $\mathbb{R} \times H_{0}^{1}(\Omega)$ whose closure links $\left(\lambda_{1} / \nu, 0\right)$ to $\left(\mu_{1} / \mu, \infty\right)$. In particular, (1.6) has at least one positive solution when $\lambda=1$ and $(\nu, \mu) \in\left\{(\nu, \mu): 0<\nu<\lambda_{1}, \mu>\mu_{1}\right\}$ or $(\nu, \mu) \in\left\{(\nu, \mu): \nu>\lambda_{1}, 0<\mu<\mu_{1}\right\}$.

This result can be seen as a complement of Theorem 3.4 of [29] where the authors considered (1.6) with $a=b=1, \lambda=1, f(x, s)=\nu s+\mu s^{3}$. They got the local existence result of positive solution when $(\nu, \mu)$ belongs to a subset of $\left\{(\nu, \mu): 0<\nu<\lambda_{1}+\varepsilon^{*}, \mu>\mu_{1}\right\}$.

\section{Answer to an open problem}

In this section, we consider problem (1.6) with $a=0$, i.e. the following problem:

$$
\begin{cases}-b \int_{\Omega}|\nabla u|^{2} d x \Delta u=\lambda f(x, u) & \text { in } \Omega, \\ u=0 & \text { on } \Omega .\end{cases}
$$

To prove Theorem 1.8, we need the following topological lemma.

Lemma 5.1. Let $\mathcal{X}$ be a normal space and let $\left\{\mathcal{C}_{n}\right\}$ be a sequence of unbounded connected subsets of $\mathcal{X}$. Assume that:

(a) there exists $z^{*} \in \liminf _{n \rightarrow+\infty} \mathcal{C}_{n}$ with $\left\|z^{*}\right\|<+\infty$;

(b) for every $R>0,\left(\bigcup_{n=1}^{+\infty} \mathcal{C}_{n}\right) \cap B_{R}$ is a relatively compact set of $\mathcal{X}$, where $B_{R}=\{x \in \mathcal{X}:\|x\| \leq R\}$.

Then $\mathcal{D}=\limsup _{n \rightarrow+\infty} \mathcal{C}_{n}$ is unbounded, closed and connected.

Proof. Let $X_{R}=\mathcal{X} \cap B_{R}$ for any $R>0$. Then $X_{R}$ is a metric subspace under the induced topology of $\mathcal{X}$. Let $A_{n}=\mathcal{C}_{n} \cap B_{R}$. Clearly, we have $\bigcup_{n=1}^{+\infty} A_{n}=$ $\left(\bigcup_{n=1}^{+\infty} \mathcal{C}_{n}\right) \cap B_{R}$. So $\bigcup_{n=1}^{+\infty} A_{n}$ is relatively compact in $X_{R}$. Furthermore, $z^{*} \in$ $\liminf _{n \rightarrow+\infty} \mathcal{C}_{n}$ implies that every neighbourhood $U\left(z^{*}\right)$ of $z^{*}$ contains points of all 
but a finite number of sets of $\left\{\mathcal{C}_{n}\right\}$. So there exists a positive integer $N$ such that for $n>N, U\left(z^{*}\right) \cap \mathcal{C}_{n} \neq \emptyset$. Since $\left\|z^{*}\right\|<+\infty$, we can take $R>0$ large enough such that $U\left(z^{*}\right) \subseteq B_{R}$. Thus, $U\left(z^{*}\right) \cap A_{n}=U\left(z^{*}\right) \cap \mathcal{C}_{n} \neq \emptyset$ for $n>N$. So we have $z^{*} \in \liminf _{n \rightarrow+\infty} A_{n}$. By Theorem 9.1 of [39], it follows that $A=\limsup _{n \rightarrow+\infty} A_{n}$ is connected in $X_{R}$.

We claim that $B:=\left(\limsup _{n \rightarrow+\infty} \mathcal{C}_{n}\right) \cap B_{R}=A$. For $x \in A$, any neighbourhood $V$ in $X_{R}$ of $x$ contains points of infinitely many sets of $\left\{\mathcal{C}_{n} \cap B_{R}\right\}$. So there exist $x_{n_{i}} \in \mathcal{C}_{n_{i}} \cap B_{R}$ such that $x_{n_{i}} \rightarrow x$ as $i \rightarrow+\infty$. It follows that $x \in B_{R}$ and $x \in \limsup _{n \rightarrow+\infty} \mathcal{C}_{n}$, i.e. $x \in B$. Conversely, if $x \in B$, any neighbourhood $V$ in $X_{R}$ of $x$ contains a point $z$ of $\limsup \mathcal{C}_{n}$ and thus $V$, a neighborhood of $z$, contains points of infinitely many sets of $\mathcal{C}_{n} \cap B_{R}$. It follows that $x \in A$. Hence, $B$ is connected. As $R$ is arbitrary, we get that $\mathcal{D}$ is connected. From [39], we know that $\mathcal{D}$ is closed.

Next, we show that $\mathcal{D}$ is unbounded. Suppose on the contrary that $\mathcal{D}$ is bounded. It is easy to see that $\mathcal{D}$ is a compact set of $\mathcal{X}$ by (b) and the fact of $z^{*} \in \mathcal{D}$. Let $U_{\delta}$ be a $\delta$-neighbourhood of $\mathcal{D}$. So we have that

$$
\partial U_{\delta} \cap \mathcal{D}=\emptyset .
$$

By (a) and the connectedness of $\mathcal{C}_{n}$, there exists an integer $N_{0}>0$ such that for all $n>N_{0}, \mathcal{C}_{n} \cap \partial U_{\delta} \neq \emptyset$. Take $y_{n} \in \mathcal{C}_{n} \cap \partial U_{\delta}$, then $\left\{y_{n}: n>N_{0}\right\}$ is a relatively compact subset of $\mathcal{X}$, so there exist $y^{*} \in \partial U_{\delta}$ and a subsequence $\left\{y_{n_{k}}\right\}$ such that $y_{n_{k}} \rightarrow y^{*}$. The definition of superior limit shows that $y^{*} \in \mathcal{D}$. Therefore, $y^{*} \in \partial U_{\delta} \cap \mathcal{D}$. However, this contradicts (5.2). Therefore, $\mathcal{D}$ is unbounded.

In order to apply Lemma 5.1 to prove Theorem 1.8, we need to discuss the following Kirchhoff-Laplace operator $\Phi_{k}(u):=-b\|u\|^{2} \Delta u$.

Denote $\Phi(u):=b\|u\|^{4} / 4$. It is obvious that the functional $\Phi$ is continuously Gâteaux differentiable and its Gâteaux derivative at the point $u \in X$ is the functional $\Phi^{\prime}(u) \in X^{*}$ given by

$$
\left\langle\Phi^{\prime}(u), v\right\rangle=b\|u\|^{2} \int_{\Omega} \nabla u \cdot \nabla v d x .
$$

Obviously, the Kirchhoff-Laplace operator is the derivative operator of $\Phi$ in the weak sense. We have the following properties of the derivative operator of $\Phi$.

Proposition 5.2. Let $L=\Phi^{\prime}$. Then:

(a) $L: X \rightarrow X^{*}$ is a continuous and strictly monotone operator;

(b) $L$ is a mapping of type $\left(\mathrm{S}_{+}\right)$, i.e. if $u_{n} \rightarrow u$ in $X$ and

$$
\varlimsup_{n \rightarrow+\infty}\left\langle L\left(u_{n}\right)-L(u), u_{n}-u\right\rangle \leq 0
$$

then $u_{n} \rightarrow u$ in $X$; 
(c) $L(u): X \rightarrow X^{*}$ is a homeomorphism.

Proof. (a) Let $u_{n} \rightarrow u$ in $X$, i.e. $\lim _{n \rightarrow+\infty}\left\|u_{n}-u\right\|=0$. Then we can easily see that $\lim _{n \rightarrow+\infty}\left\|u_{n}\right\|=\|u\|$. For any $v \in X$, by using of the Hölder inequality, we have

$$
\begin{aligned}
\mid\left\langle L\left(u_{n}\right)\right. & -L(u), v\rangle \mid \\
= & \left|b\left\|u_{n}\right\|^{2} \int_{\Omega} \nabla u_{n} \cdot \nabla v d x-b\|u\|^{2} \int_{\Omega} \nabla u \cdot \nabla v d x\right| \\
= & \left|b\left\|u_{n}\right\|^{2} \int_{\Omega}\left(\nabla u_{n}-\nabla u\right) \cdot \nabla v d x+b\left(\left\|u_{n}\right\|^{2}-\|u\|^{2}\right) \int_{\Omega} \nabla u \cdot \nabla v d x\right| \\
& \leq b\left\|u_{n}\right\|^{2}\left\|u_{n}-u\right\|\|v\|+b\left|\left\|u_{n}\right\|^{2}-\|u\|^{2}\right|\|u\|\|v\| \rightarrow 0 \text { as } n \rightarrow+\infty .
\end{aligned}
$$

It follows that $L$ is a continuous operator.

For any $u, v \in X$ with $u \neq v$ in $X$, by the Cauchy inequality, we obtain

$$
\begin{aligned}
& \langle L(u)-L(v), u-v\rangle \\
& \quad=\langle L(u), u\rangle-\langle L(u), v\rangle-\langle L(v), u\rangle+\langle L(v), v\rangle \\
& \quad=b\|u\|^{4}-b\|v\|^{2} \int_{\Omega} \nabla u \cdot \nabla v d x-b\|u\|^{2} \int_{\Omega} \nabla u \cdot \nabla v d x+b\|v\|^{4} \\
& \quad=b\left(\|u\|^{4}+\|v\|^{4}-\left(\|u\|^{2}+\|v\|^{2}\right) \int_{\Omega} \nabla u \cdot \nabla v d x\right) \\
& \quad \geq b\left(\|u\|^{4}+\|v\|^{4}-\frac{\left(\|u\|^{2}+\|v\|^{2}\right)^{2}}{2}\right)=b\left(\|u\|^{2}-\|v\|^{2}\right)^{2} \geq 0
\end{aligned}
$$

i.e. $L$ is monotone. In fact $L$ is strictly monotone. Indeed, if $\langle L(u)-L(v), u-v\rangle$ $=0$, then from (5.3) we have

$$
\|u\|=\|v\|, \quad \nabla u \equiv \nabla v \quad \text { a.e. in } \Omega .
$$

It follows that $\|u-v\|=0$, i.e. $u \equiv v$, what contradicts $u \neq v$ in $X$. Therefore, $\langle L(u)-L(v), u-v\rangle>0$. It follows that $L$ is a strictly monotone operator in $X$.

(b) From (a), if $u_{n} \rightarrow u$ and $\varlimsup_{n \rightarrow+\infty}\left\langle L\left(u_{n}\right)-L(u), u_{n}-u\right\rangle \leq 0$, then we have

$$
\lim _{n \rightarrow+\infty}\left\langle L\left(u_{n}\right)-L(u), u_{n}-u\right\rangle=0 .
$$

In view of (5.3), we obtain that $\nabla u_{n}$ converges in measure to $\nabla u$ in $\Omega$, so we get a subsequence (which we still denote by $u_{n}$ ) satisfying $\nabla u_{n}(x) \rightarrow \nabla u(x)$ for almost every $x \in \Omega$. Moreover, we also have

$$
\lim _{n \rightarrow+\infty} \int_{\Omega}\left|\nabla u_{n}\right|^{2} d x=\int_{\Omega}|\nabla u|^{2} d x .
$$

From (5.4) it follows that the integrals of the functions family $\left\{\left|\nabla u_{n}\right|^{2}\right\}$ possess absolute equi-continuity on $\Omega$ (see [34, Chapter 6 , Section 3]). Since

$$
\left|\nabla u_{n}-\nabla u\right|^{2} \leq 2\left(\left|\nabla u_{n}\right|^{2}+|\nabla u|^{2}\right),
$$


the integrals of the family $\left\{\left|\nabla u_{n}-\nabla u\right|^{2}\right\}$ are also absolutely equi-continuous on $\Omega$ and therefore we have

$$
\lim _{n \rightarrow+\infty} \int_{\Omega}\left|\nabla u_{n}-\nabla u\right|^{2} d x=0 .
$$

Therefore, $u_{n} \rightarrow u$, i.e. $L$ is of type $\left(\mathrm{S}_{+}\right)$.

(c) It is clear that $L$ is an injection. Since

$$
\lim _{\|u\| \rightarrow+\infty} \frac{\langle L(u), u\rangle}{\|u\|}=\lim _{\|u\| \rightarrow+\infty} b\|u\|^{3}=+\infty,
$$

$L$ is coercive, thus $L$ is a surjection in view of the Minty-Browder theorem (see [40, Theorem 26A]). Hence $L$ has an inverse mapping $L^{-1}: X^{*} \rightarrow X$. Therefore, the continuity of $L^{-1}$ is sufficient to ensure $L$ to be a homeomorphism.

If $f_{n}, f \in X^{*}, f_{n} \rightarrow f$, let $u_{n}=L^{-1}\left(f_{n}\right), u=L^{-1}(f)$, then $L\left(u_{n}\right)=f_{n}$, $L(u)=f$. The coercive property of $L$ implies that $\left\{u_{n}\right\}$ is bounded in $X$. We can assume that $u_{n_{k}} \rightarrow u_{0}$ in $X$. By $f_{n_{k}} \rightarrow f$ in $X^{*}$, we have

$$
\lim _{k \rightarrow+\infty}\left\langle L\left(u_{n_{k}}\right)-L\left(u_{0}\right), u_{n_{k}}-u_{0}\right\rangle=\lim _{n \rightarrow+\infty}\left\langle f_{n_{k}}, u_{n_{k}}-u_{0}\right\rangle=0 .
$$

Since $L$ is of type $\left(\mathrm{S}_{+}\right), u_{n_{k}} \rightarrow u_{0}$. Furthermore, the continuity of $L$ implies that $L\left(u_{0}\right)=L(u)$. By injectivity of $L$, we have $u_{0}=u$. So $u_{n_{k}} \rightarrow u$. We claim that $u_{n} \rightarrow u$ in $X$. Otherwise, there would exist a subsequence $\left\{u_{m_{j}}\right\}$ of $\left\{u_{n}\right\}$ in $X$ and $\varepsilon_{0}>0$ such that for any $j \in \mathbb{N}$, we have $\left\|u_{m_{j}}-u\right\| \geq \varepsilon_{0}$. But reasoning as above, $\left\{u_{m_{j}}\right\}$ would contain a further subsequence $u_{m_{j_{l}}} \rightarrow u$ in $X$ as $l \rightarrow+\infty$, which is a contradiction to $\left\|u_{m_{j_{l}}}-u\right\| \geq \varepsilon_{0}$. Therefore, $L^{-1}$ is continuous.

Now, consider the following auxiliary problem:

$$
\begin{cases}-b\|u\|^{2} \Delta u=f(x) & \text { in } \Omega, \\ u=0 & \text { on } \partial \Omega .\end{cases}
$$

Lemma 5.3. For any $f \in X^{*}$, problem (5.5) has a unique weak solution.

Proof. Define $\mathcal{F}(v)=\int_{\Omega} f v d x$ for any $v \in X$. We can easily see that $\mathcal{F}$ is a continuous linear functional on $X$. Since $L$ is a homeomorphism, (5.5) has a unique solution.

Let us denote by $S(f)$ the unique weak solution of (5.5). Then $S: X^{*} \rightarrow X$ is a continuous operator. Since the embedding of $X \hookrightarrow L^{q}(\Omega)$ is compact for each $q \in\left(1,2^{*}\right), S: L^{q^{\prime}}(\Omega) \rightarrow X$ is a completely operator.

For any $n \in \mathbb{N}$, we study the following auxiliary problem:

$$
\begin{cases}-\left(\frac{1}{n}+b \int_{\Omega}|\nabla u|^{2} d x\right) \Delta u=\lambda f(x, u) & \text { in } \Omega \\ u=0 & \text { on } \Omega .\end{cases}
$$


Clearly, we can see that $f_{0}=\widetilde{f}_{0} n$. Theorem 1.3 implies that there exists a sequence of unbounded continua $\mathcal{C}_{n}$ of positive solutions to problem (5.6) emanating from $\left(1 / \widetilde{f}_{0} n, 0\right)$ and joining to $\left(1 / f_{\infty},+\infty\right)$.

Proof of Theorem 1.8. Let $\mathcal{X}=\mathbb{R} \times X$ under the product topology. Clearly, $\mathcal{X}$ is a Banach space. Now, we verify the assumptions of Lemma 5.1. Taking $z_{n}=\left(1 / \widetilde{f}_{0} n, 0\right)$ and $z^{*}=(0,0)$, we have that $z^{*} \in \liminf _{n \rightarrow+\infty} \mathcal{C}_{n}$. So (a) is satisfied.

By (f3) and (f4), $F(x, u(x)) \in L^{4 / 3}(\Omega)$. Now, we consider two possibilities to verify (b): (1) $n<+\infty$ and (2) $n=+\infty$. If case (1) occurs, the completely continuous property of $G$ implies (b). If case (2) occurs, the compactness of $S$ follows (b). By Lemma 5.1, $\mathcal{C}:=\lim \sup _{n \rightarrow+\infty} \mathcal{C}_{n}$ is an unbounded component such that $(0,0) \in \mathcal{C}$ and $\left(1 / f_{\infty},+\infty\right) \in \mathcal{C}$. This completes the proof.

Corollary 5.4. Assume that $N \leq 3, \Omega$ satisfies one of the conditions in Theorem $1.2(\mathrm{c})$ and $f$ satisfies (f1), (f3) and (f4). Then for $\lambda \in\left(0,1 / f_{\infty}\right)$, problem (5.1) possesses at least one positive solution. In particularly, if $f_{\infty}<1$ then (5.1) possesses at least one positive solution with $\lambda=1$.

REMARK 5.5. From Remark 1.7, we know that any weak solution of (5.6) belongs to $C^{1, \alpha}(\bar{\Omega})$ with $\alpha \in(0,1)$ under assumptions of (f1), (f3) and (f4). It follows that $u \in C^{1, \alpha}(\bar{\Omega})$ with $\alpha \in(0,1)$ for any $(\lambda, u) \in \mathcal{C}$ which is obtained in Theorem 1.8 .

\section{Future work}

In future we plan:

(1) To study the case $f_{0} \notin(0,+\infty)\left(\widetilde{f}_{0} \notin(0,+\infty)\right)$ or $f_{\infty} \notin(0,+\infty)$. This plan origins from Theorem 1.2 and the following special example:

$$
\begin{cases}-\left(a+b \int_{\Omega}|\nabla u|^{2} d x\right) \Delta u=\lambda u & \text { in } \Omega, \\ u=0 & \text { on } \Omega .\end{cases}
$$

It is easy to see that $f_{\infty}=0$. While, the positive solution pairs of (6.1) must be $\left(\lambda_{1}\left(a+b\left\|\varphi_{1}\right\|^{2}\right), \varphi_{1}\right)$, where $\varphi_{1}$ is the corresponding positive eigenfunction to $\lambda_{1}$. Thus $\left(\lambda_{1}, 0\right)$ is a bifurcation point of the set of positive solutions of (6.1) and $\left(\lambda_{1}\left(a+b\left\|\varphi_{1}\right\|^{2}\right), \varphi_{1}\right)$ is the corresponding unique unbounded branch.

(2) To study the unilateral global bifurcation phenomenon and the existence of one-sign and sign-changing solutions for (1.1).

\section{REFERENCES}

[1] W. Allegretto and Y.X. Huang, A Picone's identity for the p-Laplacian and applications, Nonlinear Anal. 32 (1998), 819-830. 
[2] A. Arosio and S. Pannizi, On the well-posedness of the Kirchhoff string, Trans. Amer. Math. Soc. 348 (1996), 305-330.

[3] G. Autuori, P. Pucci and M.C. Salvatori, Asymptotic stability for anisotropic Kirchhoff systems, J. Math. Anal. Appl. 352 (2009), 149-165.

[4] M.M. Cavalcante, V.N. Cavalcante and J.A. Soriano, Global existence and uniform decay rates for the Kirchhoff-Carrier equation with nonlinear dissipation, Adv. Differential Equations 6 (2001), 701-730.

[5] C. Chen, Y. Kuo And T. Wu, The Nehari manifold for a Kirchhoff type problem involving sign-changing weight functions, J. Differential Equations 250 (4) (2011), 1876-1908.

[6] B. Cheng And X. Wu, Existence results of positive solutions of Kirchhoff type problems, Nonlinear Anal. 71 (10) (2009), 4883-4892.

[7] F.J.S.A. CorrêA And G.M. Figueiredo, On a elliptic equation of p-Kirchhoff type via variational methods, Bull. Austral. Math. Soc. 74 (2006), 263-277.

[8] G. Dai and R. HaO, Existence of solutions for a p(x)-Kirchhoff-type equation, J. Math. Anal. Appl. 359 (2009), 275-284.

[9] G. DAI AND D. LIU, Infinitely many positive solutions for a $p(x)$-Kirchhoff-type equation, J. Math. Anal. Appl. 359 (2009), 704-710.

[10] G. Dai AND R. MA, Solutions for a $p(x)$-Kirchhoff type equation with Neumann boundary data, Nonlinear Anal. Real World Appl. 12 (2011), 2666-2680.

[11] G. DAI AND J. WeI, Infinitely many non-negative solutions for a $p(x)$-Kirchhoff-type problem with Dirichlet boundary condition, Nonlinear Anal. 73 (2010), 3420-3430.

[12] L. Damascelli, M. Grossi and F. Pacella, Qualitative properties of positive solutions of semilinear elliptic equations in symmetric domains via the maximum principle, Ann. Inst. H. Poincaré Anal. Non Linéaire 16 (1999), 631-652.

[13] E.N. DANCER, The effect of domain shape on the number of positive solu nonlinear equations, J. Differential Equations 74 (1988), 120-156.

[14] P. D'Ancona And Y. Shibata, On global solvability of nonlinear viscoelastic equations in the analytic category, Math. Methods Appl. Sci. 17 (6) (1994), 477-486.

[15] P. D'Ancona and S. Spagnolo, Global solvability for the degenerate Kirchhoff equation with real analytic data, Invent. Math. 108 (1992), 247-262.

[16] M. Del Pino And R. Manásevich, Global bifurcation from the eigenvalues of the pLapiacian, J. Differential Equations 92 (1991), 226-251.

[17] M. Drener, The Kirchhoff equation for the p-Laplacian, Rend. Semin. Mat. Univ. Politec. Torino 64 (2006), 217-238.

[18] _ The ware equation for the p-Laplacian, Hokkaido Math. J. 36 (2007), 21-52.

[19] L.C. Evans, Partial differential equations, AMS, Rhode Island, 1998.

[20] X.L. Fan, On nonlocal p(x)-Laplacian Dirichlet problems, Nonlinear Anal. 72 (2010), $3314-3323$.

[21] _ Global $C^{1, \alpha}$ regularity for variable exponent elliptic equations in divergence form, J. Differential Equations 235 (2007), 397-417.

[22] X.L. Fan and D. ZhaO, A class of De Giorgi type and Hölder continuity, Nonlinear Anal. 36 (1996), 295-318.

[23] X.L. FAn, Y.Z. ZhaO AND Q.H. Zhang, A strong maximum principle for $p(x)$-Laplace equations, Chinese J. Contemp. Math. 24 (3) (2003), 277-282.

[24] B. Gidas, W.M. Ni And L. Nirenberg, Symmetry and related properties via the maximum principle, Comm. Math. Phys. 68 (1979), 209-243.

[25] D. Gilbarg And N.S. Trudinger, Elliptic Partial Differential Equations of Second Order, Springer, Berlin, 2001. 
[26] X. HE AND W. Zou, Infinitely many positive solutions for Kirchhoff-type problems, Nonlinear Anal. 70 (2009), 1407-1414.

[27] G. Kirchhoff, Mechanik, Teubner, Leipzig, 1883.

[28] Z. Liang, F. Li And J. Shi, Positive solutions to Kirchhoff type equations with nonlinearity having prescribed asymptotic behavior, Ann. Inst. H. Poncaré Anal, Nonlinéaire 31 (2014), 155-167.

[29] _ Positive solutions of Kirchhoff type nonlocal elliptic equation: a bifurcation approach, in press.

[30] C.S. LIN, Uniqueness of least energy solutions to a semilinear elliptic equation in $\mathbb{R}^{2}$, Manuscripta Math. 84 (1994), 13-19.

[31] J.L. Lions, On some equations in boundary value problems of mathematical physics, in: Contemporary Developments in Continuum Mechanics and Partial Differential Equations (Proc. Internat. Sympos., Inst. Mat. Univ. Fed. Rio de Janeiro, Rio de Janeiro, 1977), North-Holland Math. Stud., vol. 30, North-Holland, Amsterdam, 1978, pp. 284-346.

[32] T.F. Ma And J.E.Muñoz Rivera, Positive solutions for a nonlinear nonlocal elliptic transmission problem, Appl. Math. Lett. 16 (2) (2003), 243-248.

[33] A. MAO AND Z. ZhANG, Sign-changing and multiple solutions of Kirchhoff type problems without the P.S. condition, Nonlinear Anal. 70 (3) (2009), 1275-1287.

[34] I.P. Natanson, Theory of Functions of a Real Variable, Nauka, Moscow, 1950.

[35] K. Perera And Z. Zhang, Nontrivial solutions of Kirchhoff-type problems via the Yang index, J. Differential Equations 221 (1) (2006), 246-255.

[36] P.H. Rabinowitz, Some aspects of nonlinear eigenvalue problems, Rocky Mountain J. Math. 3 (2) (1973), 161-202.

[37] _ Some global results for nonlinear eigenvalue problems, J. Funct. Anal. 7 (1971), $487-513$.

[38] J. Sun AND C. TANG, Existence and multiplicity of solutions for Kirchhoff type equations, Nonlinear Anal. 74 (4) (2011), 1212-1222.

[39] G.T. Whyburn, Topological Analysis, Princeton University Press, Princeton, 1958.

[40] E. ZeIDLER, Nonlinear functional analysis and its applications, Vol. II/B. BerlinHeidelberg-New York 1985

[41] Z. Zhang AND K. PererA, Sign changing solutions of Kirchhoff type problems via invariant sets of descent flow, J. Math. Anal. Appl. 317 (2) (2006), 456-463.

GuOWei DAI

School of Mathematical Sciences

Dalian University of Technology

Dalian, 116024, P.R. CHINA

E-mail address: daiguowei@dlut.edu.cn 